\title{
Cross-Linked Alginate Film Pore Size Determination Using Atomic Force Microscopy and Validation Using Diffusivity Determinations
}

\author{
Cheryl Simpliciano ${ }^{1}$, Larissa Clark ${ }^{1}$, Behrokh Asi ${ }^{1}$, Nathan $\mathrm{Chu}^{1}$, Maria Mercado ${ }^{1}$, Steven Diaz $^{1}$, \\ Michel Goedert $^{1 \#}$, Maryam Mobed-Miremadi ${ }^{1,2 \#}$
}

\author{
${ }^{1}$ Department of Biomedical, Chemical and Materials Engineering, San Jose State University, San Jose, USA; ${ }^{2}$ Department of Bioen- \\ gineering, Santa Clara University, Santa Clara, USA. \\ Email: "michel.goedert@sjsu.edu, "mmobedmiremadi@scu.edu
}

Received July $4^{\text {th }}, 2013$; revised August $6^{\text {th }}, 2013$; accepted September $1^{\text {st }}, 2013$

Copyright (C) 2013 Cheryl Simpliciano et al. This is an open access article distributed under the Creative Commons Attribution License, which permits unrestricted use, distribution, and reproduction in any medium, provided the original work is properly cited.

\begin{abstract}
The deficit of organ donors has fueled the need for advances in tissue engineering and regenerative medicine. Microencapsulation in alginate immuno-isolation membranes has been used to treat many disabling metabolic disorders, namely, phenylketonuria, kidney failure and diabetes mellitus. Systematic nutrient flux determinations are hindered by the lack of experimental data on alginate-based membrane topography and the pore size thus preventing the full therapeutic potential of the bio-membranes to be reached. In this study, samples of cross-linked alginate membranes were subjected to the following analytical characterization: 1) pore size characterization using atomic force microscopy operated in contact mode to detect and measure pore size; 2) differential scanning calorimetry to confirm biopolymer cross-linking; and 3 ) diffusivity measurements using spectrophotometry and fluorescence microscopy to confirm the presence of through pores and to calculate reflection coefficients. The pore sizes for the pre-clinical standard formulation of $1.5 \%(\mathrm{w} / \mathrm{v}) \mathrm{me}-$ dium viscosity alginate cross-linked with $1.5 \% \mathrm{CaCl}_{2}$ and $0.5 \%(\mathrm{w} / \mathrm{v})$ alginate and chitosan cross-linked with $20 \%$ $\mathrm{CaCl}_{2}$ are $5.2 \mathrm{~nm} \pm 0.9 \mathrm{~nm}$ and $7.0 \mathrm{~nm} \pm 3.1 \mathrm{~nm}$, respectively. An increase in the glass transition temperatures as a function of cross-linker concentration was observed. Diffusivity values obtained from the inward diffusivity of creatinine into macrocapsules $(\mathrm{d}=1000 \mu \mathrm{m} \pm 75 \mu \mathrm{m})$ and the outward diffusivity of FITC dextrans from macrocapsules $(\mathrm{d}=1000 \mu \mathrm{m} \pm 75 \mu \mathrm{m})$ and microcapsules $(\mathrm{d}=40 \mu \mathrm{m} \pm 5 \mu \mathrm{m})$ were shown to correlate strongly $\left(\mathrm{R}^{2}=0.9835\right)$ with the ratio of solute to pore sizes, confirming the presence of through pores. Reflection coefficients approaching and exceeding unity correlate with the lack of permeability of the membranes to MW markers that are $70 \mathrm{kDa}$ and greater.
\end{abstract}

Keywords: Alginate; Atomic Force Microscopy; Pore Size; Stokes’ Radius; Diffusivity; Cross-linking; Differential Scanning Calorimetry; Reflection Coefficient

\section{Introduction}

Novel therapies resulting from regenerative medicine and tissue engineering technology may offer a new hope for patients with injuries, metabolic disorders, cancer, and end-stage organ failure. As an example, currently, patients with diseased and injured organs are often treated with transplanted organs. However, there is a shortage of donor organs that is worsening yearly as the population ages and as the number of new cases of organ failure

\footnotetext{
"Declaration of interest: Authors have no declaration of interests to report.

${ }^{\#}$ Corresponding authors.
}

increases [1]. Bio-printing, including microencapsulation of cells, enzymes and drugs in biocompatible hydrogels, has been researched in an organ prototyping and metabolic disorders [2,3], stem cell encapsulation [4] and cancer [5]. This use of hydrogels can be attributed to the ability of the hydrogel to form a biodegradable and biocompatible encapsulation matrix once cross-linked [6]. The most common hydrogel biopolymer used in transplantation and cell therapy is alginate [2].

Alginate is a naturally-occurring, water-soluble polymer comprised of $(1,4)$-linked $\beta$-D-mannuronic (M) and $(1,3)-\alpha$-L-guluronic $(\mathrm{G})$ acid residues. Different varieties of alginate contain varying ratios of M and G. Depending 
on the arrangement of the varying M, G, or MG blocks, alginate copolymers of slightly different behaviors and properties can be produced. Alginate can be gently cross-linked by the addition of divalent cations [7]. The G-block is stiffer and more extended in chain configuration than the M-block due to a higher degree of hindered rotation around the glycosidic linkages [8]. The removal of the "M" residues, constituting a significant portion of the alginate polymer, has increased biocompatibility by many folds [9]. The substitution of calcium by barium as the cross-linking divalent ion [10] and the use of chitosan/ genipin-chitosan alginate membranes [11] have resulted in tremendous improvements in membrane strength. A significant amount of research and development has been dedicated to the reproducible molding of cross-linked alginate membrane into microfibers [12], high-throughput microcapsule miniaturization [13] and transdermal patches [14].

The gelation of alginate is possible by interaction of carboxylate groups with divalent ions, namely, calcium [15]. The outcome of the gelation process and hence the pore size can be modulated by using alginates of different molecular weight and concentrations [16] and alginates comprised of different amounts of $\mathrm{G}$ fractions [17], modulating the crosslinker concentration and/or cross-linking reaction time [18] and by combining interactions of all of these factors.

The molecular weight cutoff (MWCO) of the membrane expressed in terms of Stokes' radius, (a), is the maximum molecular weight that is allowed through the selective passage of the membrane pores given by Equation (1) [19]. This equation assumes that the solute of molecular weight $(\mathrm{MW})$ is a sphere with a density $(\rho=1$ $\mathrm{g} \cdot \mathrm{cm}^{-3}$ ) equal to that of the solute in solid phase. The pore sizes in the gel network of hydrogels vary from macroporous $(0.1-1 \mu \mathrm{m})$ to microporous $(10-100 \mathrm{~nm})$ [20]. Shown in Figure 1 is a cross-section of an alginate microcapsule captured by SEM.

$$
a=\left(\frac{3 \mathrm{MW}}{4 \pi \rho \mathrm{N}_{\mathrm{A}}}\right)^{1 / 3}
$$

The pore size of an encapsulation material is critical to both encapsulation efficiency and release kinetics. Too large of a pore size will allow content leakage while too small of a pore size can hinder timely release. Alginate pore size has been extensively researched through various techniques, mainly through imaging and diffusivity measurements. However, there is little agreement as to what the pore sizes actually are. Tabulated results indicating the variation in pore sizes appear in Table 1. The reported pore sizes apply to either alginate films or microcapsules. As shown by results of diffusion studies, alginate pores can range from $3.6-14 \mathrm{~nm}$ for $4 \%$ alginate $[21,22]$ and $3 \mathrm{~nm}$ and $14.5-17 \mathrm{~nm}$ for $1.5 \%$ and $3 \%$ alginate, respectively [23]. In experiments where scanning electron micros-

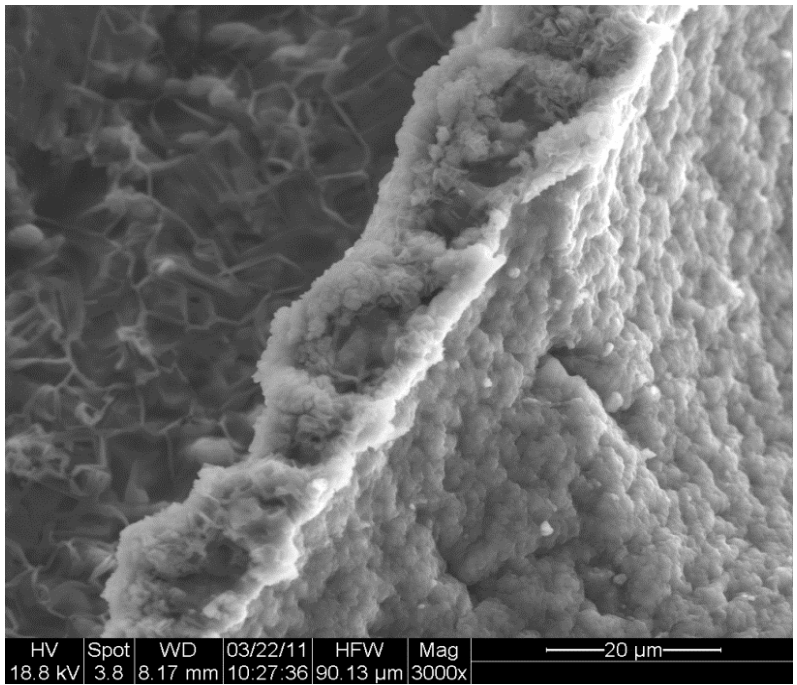

Figure 1. SEM image of $0.5 \% \mathrm{MV}$ alginate/20\% $\mathrm{CaCl}_{2}$ microcapsule cross section, dehydrated. Captured in low-vacuum mode.

copy (SEM) was used, a larger range of pore sizes from 5 $\mathrm{nm}-21 \mu \mathrm{m}$ have been observed $[7,15,24,25]$. Numerous atomic force microscopy (AFM) imaging experiments produced pore sizes between $10 \mathrm{~nm}$ and $1.3 \mu \mathrm{m}$ [10,26,27]. Pore sizes less than $10 \mathrm{~nm}$ and as large as $70 \mathrm{~nm}$ were revealed using Transmission Electron Microscopy (TEM) in experiments conducted by Leal-Egaña, Braumann, DiazCuenca, Nowicki and Bader [28]. A maximum pore size of $5.8 \mathrm{~nm}$ was obtained, based on fluorescent microscopy measurements [29]. Sources of discrepancies include the range of variables associated with the gelation technique, the artifacts of sample preparation, and the resolution of the measurement technique.

In the absence of precise pore size data, systematic flux determinations are hindered by the lack of experimental data on membrane topography, thus preventing the full therapeutic potential of the alginate immuno-isolation membranes to be reached. The research objectives of this study are three-fold: 1) to measure the pore size of various alginate formulations using AFM; 2) to confirm the occurrence of cross-linking using differential scanning calorimetry (DSC); and 3) to correlate measured pore sizes to diffusivity measurements. Of particular interest are the pore sizes for the pre-clinical standard formulation of $1.5 \%(\mathrm{w} / \mathrm{v})$ alginate cross-linked with $1.5 \% \mathrm{CaCl}_{2}[2]$ and the MWCO of the miniaturized capsule membrane, $0.5 \%(\mathrm{w} / \mathrm{v})$ alginate/chitosan cross-linked with $20 \% \mathrm{CaCl}_{2}$, characterized by faster toxin clearance in-vitro [30].

\section{Materials and Methods}

\subsection{Materials}

All chemicals used in this study were acquired from 
Microscopy and Validation Using Diffusivity Determinations

Table 1. Literature review of pore size for various analytical methods.

\begin{tabular}{|c|c|c|c|c|}
\hline Study & Method & $\begin{array}{l}\text { Wet/Dry Imaging } \\
\text { Conditions }\end{array}$ & Membrane Morphology/Type & Pore Size \\
\hline Wang, et al. [7] & Cryo-SEM & Dry & Microcapsules (Calcium Chloride) & $3.9-10.9 \mu \mathrm{m}$ \\
\hline Zimmerman, et al. [10] & AFM & Wet & Thick film (Barium Chloride) & $1.2-1.3 \mu \mathrm{m}$ \\
\hline Gombotz and Wee [15] & SEM & Dry & Microcapsules (Calcium Chloride) & $5-200 \mathrm{~nm}$ \\
\hline Choi, et al. [21] & Diffusion & Wet & Microfluidic scaffold (Calcium Chloride) & $3.6 \mathrm{~nm}$ \\
\hline Chan and Neufeld [22] & Diffusion & Wet & Microcapsules (Calcium Chloride) & $4-14 \mathrm{~nm}$ \\
\hline $\mathrm{Li}$, et al. [23] & Diffusion & Wet & Cylinders (Calcium Chloride) & $14.5-17 \mathrm{~nm}$ \\
\hline Wright, et al. [24] & SEM & Dry & Slabs (Calcium Chloride) & $0.1-0.3 \mu \mathrm{m}$ \\
\hline Jejurikar, et al. [25] & Cryo-SEM & Dry & $\begin{array}{l}\text { Low Viscosity Alginate Films } \\
\text { (Calcium Chloride and Barium Chloride) }\end{array}$ & $0.5-21 \mathrm{um}$ \\
\hline Hsiong, et al. [26] & AFM & Dry & Films (Calcium Chloride) & $10-100 \mathrm{~nm}$ \\
\hline Schmid, et al. [27] & AFM & Wet & Films (Calcium Chloride) & $50-300 \mathrm{~nm}$ \\
\hline Leal-Egaña, et al. [28] & TEM & & Microcapsules (Glutaraldehyde) & $10-70 \mathrm{~nm}$ \\
\hline Mobed-Miremadi, et al. [29] & Fluorescence Microscopy & Wet & Artificial Cells (Calcium Chloride) & $<5.8 \mathrm{~nm}$ \\
\hline
\end{tabular}

Sigma-Aldrich (USA); these are: medium molecular weight (MV) sodium-alginate (A2033), low molecular weight (LV) sodium-alginate (A2158), low molecular weight chitosan (44 886-9, 75\% deacetylated, 3.8-6 kDa) and fluorescein isothiocyanate dextran markers abbreviated as FITC Dextran markers (46947, FD70S, FD4). All other reagent grade chemicals were provided by the Chemistry store in the Faculty of Sciences at San Jose State University: creatinine powder $(\mathrm{MW}=113 \mathrm{Da})$ and bovine serum albumin (BSA, MW $=66.4 \mathrm{kDa})$. The triangular Pyrex-Nitride AFM probes (PNP-TR-20) were purchased from NanoWorld (Neuchâtel, Switzerland). Polylysine-coated slides were purchased from VWR (Radnor, PA) (cat\# 16002-116). Cellulose Ester (CE) dialysis tubing with a molecular weight a cutoff (MWCO) of $20 \mathrm{kDa}$ was puchased from Spectrum Labs (SpectraPor \# 131342, Rancho Dominguez, CA).

\subsection{Methods}

It should be noted that it was not possible to use a single type of alginate structure for all analytical tests. While films were used for AFM and differential scanning calorimetry (DSC), spherical capsules were used for diffusivity measurements. Due to the approximate average ratio of AFM scan area to capsule area $\left(1: 10^{5}\right)$, it has been assumed that the sphere curvature can be neglected and thus the pore sizes for the spherical and flat structures are nterchangeable for the same formulation.

\subsection{Atomic Force Microscopy (AFM)}

\subsubsection{Sample Preparation}

AFM imaging was performed on spin-coated films prepared with various alginate and cross-linker concentrations and the dialysis tubing standard. Samples were cast as films for ease of imaging. $1 \mathrm{~mL}$ of alginate dissolved in saline $(0.9 \% \mathrm{NaCl} \mathrm{w} / \mathrm{v})$ at a given concentration was deposited onto a poly-L-Lysine (PLL)-coated glass slide placed into a small petri dish. The dish was fixed to a homemade spin coater comprised of a CPU fan attached to a power supply shown in Figure 2. The alginate was allowed to spin for 15 s producing a film with a relatively homogenous thickness. Calcium chloride at a given concentration was added to the film in a drop-wise fashion to induce cross-linking. The films were set to cross-link for $1 \mathrm{hr}$ followed by a DI water rinse. The PLL-coated slide was then transferred directly onto the AFM platform for imaging.

\subsubsection{Measurements}

Surface imaging was performed on the various alginate film formulations. The characterization was conducted using an Agilent 5500 AFM equipped with a contactmode nose amplifier and $100 \mu \mathrm{m}$ scanner N0524A (Agilent, Santa Clara, CA). Calibrations were performed using a TGZ02 standard (MikroMasch, Wilsonville, OR) and dialysis tubing with a MWCO of $20 \mathrm{kDa}$. The instrument was operated in contact mode using a Pyrex- 


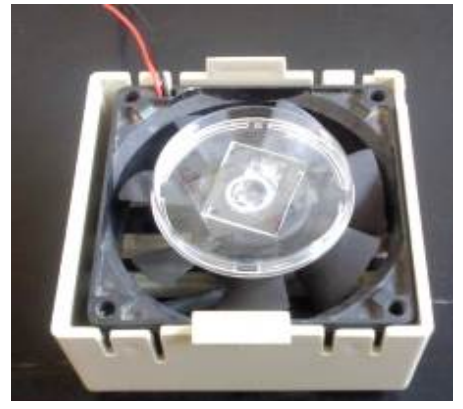

Figure 2. Spin coater/fan set up for AFM sample preparation.

Nitride probe with triangular cantilever (resonant frequency $17 \mathrm{kHz}$, force constant $0.08 \mathrm{~N} / \mathrm{m}$, thickness 600 $\mathrm{nm}$, length $200 \mu \mathrm{m}$, tip radius $7-10 \mathrm{~nm}$ ). PicoView v1.8 (Agilent, Santa Clara, CA) and Gwyddion v2.3 (Czech Metrology Institute, Brno, Czechoslovakia) were used as qualitative real-time and quantitative image analysis software, respectively. Scan speed was established by setting a ratio of 128 pixels/line. The scan area ranged from 0.1 to $5.0 \mu \mathrm{m}^{2}$ with a maximum possible range of $100 \mu \mathrm{m}$. The pore size was obtained by measuring the distance between the darkest areas of the pores as indicated by the grayscale intensity in Gwyddion. Images were obtained at different locations around the sample. These locations were changed through the movement of the stage to obtain an average pore size.

\subsection{Differential Scanning Calorimetry (DSC)}

\subsubsection{Sample Preparation}

DSC testing required samples that were no more than 5 $\mathrm{mm}$ in thickness and preferably relatively flat. $10 \mathrm{~mL}$ of alginate was spread into glass dishes. Each sample was immersed in a solution of $\mathrm{CaCl}_{2}$ of concentrations ranging from $10 \%$ to $25 \%$, allowed to cross-link for one hour and turned over once to ensure uniform cross-linking. An uncross-linked reference sample of bare alginate was also tested as control. As described by Russo, Malinconico and Santagata. [17], water may effectively mask the relatively weak glass transition shoulder of alginate in a DSC thermograph. In response, sheets of hydrated sample material were desiccated in a dry nitrogen box for a minimum period of 24 hours prior to testing. Circular segments were die-cut from the desiccated sheets, weighed, and sealed in high-purity aluminum crucibles. Specimen masses varied between 6 and $12 \mathrm{mg}$. All films were comprised of an alginate concentration of $1.5 \%(\mathrm{w} / \mathrm{v}) \mathrm{MV}$ alginate. As previously stated, this is the nominal reported molecular weight and concentration for cell encapsulation [2].

\subsubsection{Measurements}

All testing was performed by BAE Systems in Santa
Clara, CA, using a Mettler-Toledo DSC823e differential scanning calorimeter (DSC). Temperature and heat flow calibration were performed using NIST reference In, Hg and $\mathrm{Zn}$. All specimens were tested for glass transition temperature characterization by DSC over a range of 30 to $200^{\circ} \mathrm{C}$ at a rate of $10^{\circ} \mathrm{C} / \mathrm{min}$. Dry nitrogen was used to purge the sample chamber at a flow rate of $40 \mathrm{~mL} / \mathrm{min}$. The glass transition region of each thermograph was evaluated per ASTM E1356. The midpoint temperature, or the half-way point between upper and lower baselines, was reported at the glass transition temperature $(\mathrm{Tg})$ in each case.

\subsection{Macrocapsule Preparation}

Macrocapsules (MA) were fabricated using the atomization method [31]. A 1.5\% MV sodium-alginate solution was jetted into a $1.5 \%(\mathrm{w} / \mathrm{v}) \mathrm{CaCl}_{2}$ bath. The air (FA) and liquid (FL) flow rates were adjusted to $1.5 \mathrm{~L} / \mathrm{min}$ and $0.5 \mathrm{~mL} / \mathrm{min}$, respectively. After jetting, capsules were allowed to cross-link in the $\mathrm{CaCl}_{2}$ solution for $1 \mathrm{hr}$. The calcified sodium-alginate beads were then washed with $0.9 \% \mathrm{NaCl}$ twice.

\subsection{Microcapsule Preparation}

Microcapsules (MI) were fabricated using Microfab's Jetlab System using the methodology in reference [32]. The inkjet engine fires the $0.5 \% \mathrm{LV}$ sodium alginate solution through the print head into a $20 \%(\mathrm{w} / \mathrm{v}) \mathrm{CaCl}_{2}$ solution. After jetting, capsules were allowed to crosslink in the $\mathrm{CaCl}_{2}$ solution for $30 \mathrm{~min}$. Then $1 \%(\mathrm{w} / \mathrm{v})$ chitosan was added into the $20 \% \mathrm{CaCl}_{2}$ solution to make the final chitosan concentration $0.5 \%(\mathrm{w} / \mathrm{v})$. Capsules were coated for an additional $30 \mathrm{~min}$. Next, the capsules were centrifuged at $8000 \mathrm{~g}$ for $5 \mathrm{~min}$ and washed with a $0.9 \%(\mathrm{w} / \mathrm{v}) \mathrm{NaCl}$ solution 3 times.

\subsection{Diffusivity Measurements and Modeling}

\subsubsection{Creatinine}

Creatinine was used as test solute to determine inward diffusivity coefficients through MA according to previously established methodology [33]. Calibration stock solutions ranging from 0 to $5 \mathrm{mg} / \mathrm{mL}$ were used. In this range, there was a linear relationship between absorbance and concentration that was subsequently used for concentration interpolation. A $5 \mathrm{~mL}$ suspension of microcapsules was poured into a $5 \mathrm{~mL}$ solution of solute at an initial concentration $\mathrm{C}_{0}$. The objective was to measure the amount of solute diffusing from the solution through the empty MA membrane. Every $30 \mathrm{~s}$, the supernatant was tested for a change in solute concentration by measuring the absorbance at $\lambda=265 \mathrm{~nm}$ for creatinine using an Agilent 8453 UV-VIS spectrophotometer. Sampling 
stopped when no more absorbance/concentration changes were detected $(\mathrm{dC} / \mathrm{dt}=0)$. This concentration was taken as the equilibrium concentration (Ceq).

\subsubsection{FITC Dextrans}

FITC dextrans were used as test solutes to determine outward diffusivity coefficients through MA and MI according to previously established methodology [34]. Spectrophotometric methods were not sensitive enough to detect transport across the MI membrane, therefore, fluorescence microscopy was used. Calibration stock solutions ranging from 0.1 to $15.1 \mathrm{mg} / \mathrm{mL}$ for each FITCDextran MW standard (4, 70, and $500 \mathrm{kDa}$ dissolved in $0.9 \%(\mathrm{w} / \mathrm{v}) \mathrm{NaCl})$ were prepared. In this range, for each FITC-Dextran MW, there was a linear relationship between intensity and the concentration of the fluorescent marker under observation, the results of which were subsequently used for concentration interpolation. $10 \mu \mathrm{L}$ of MI or MA solution were incubated in $1 \mathrm{~mL}$ of FITC solution for $24 \mathrm{~h}$ prior to imaging. The solution was centrifuged at $8000 \mathrm{~g}$ for $5 \mathrm{~min}$ and washed once with a $0.9 \%(\mathrm{w} / \mathrm{v}) \mathrm{NaCl}$ solution. Samples were then deposited onto a microscope slide and observed under the transmission microscope/camera (Nikon EclipseTi-S/Andor Technology Interline CCD camera). The FITC/Acridine Orange filter was chosen from the imaging software (NISElements v.3.2.2) filter selection feature to accommodate the excitation and emission wavelengths of 468 and 520 $\mathrm{nm}$ of the FITC molecule. Images were captured every $30 \mathrm{~s}$. Sampling stopped when no more intensity changes were detected $(\mathrm{dI} / \mathrm{dt}=0)$. This concentration was taken as the equilibrium concentration (Ceq).

\subsubsection{Diffusivity Modeling and Calculation of the Sieving Coefficient}

The analytical solution to Fick's second law in spherical coordinates was used to determine diffusivity coefficients from spectrophotometric and fluorescence measurements according to previously published methodology [29]. Residual sum of squares (RSS) minimization was conducted using MATLAB 2010a. The membrane reflection coefficient $(\sigma)$ was calculated using Equation (2), where $\lambda$ is the ratio of the solute Stokes' radius $(\alpha)$ and the average membrane pore size $(r)$ [19].

$$
\sigma=1-(1-\lambda)^{2}\left[2-(1-\lambda)^{2}\right]\left[1-\frac{2}{3} \lambda^{2}-0.163 \lambda^{3}\right]
$$

\section{Results}

\subsection{Atomic Force Microscopy}

As previously stated, AFM imaging was performed on spin-coated films prepared with varying concentrations of alginate and $\mathrm{CaCl}_{2}$ with and without a chitosan coating.
Prior to imaging, the samples were slightly hydrated by a DI water rinse. AFM was chosen for imaging as it is an imaging method that provides nanometer resolution and three-dimensional surface imaging, requires minimal sample preparation and allows imaging in ambient and liquid conditions.

In Figure 3, the apparent variation in pore size due to the increase in resolution is plotted as a function of scan area by film formulation. A decrease in average pore size is observed across all formulations with decreasing scan area. The calculated Stokes' radius corresponding to a MWCO of $20 \mathrm{kDa}$ for the standard dialysis tubing is 2.02 $\mathrm{nm}$ [19]. For an AFM scan area of $0.1 \mu^{2}$, an average pore diameter of $4.9 \mathrm{~nm}$ was obtained for the standard sample. This value is the closest to the theoretical Stokes' radius of $2.02 \mathrm{~nm}$ corresponding to a relative measurement error of $16.7 \%$. Hence all subsequent analyses and comparisons will be conducted for the pore sizes obtained at this setting. Pore size measurements conducted using all scan areas are presented in Table 2. Shown in Figures 4-8 are the corresponding 2D views for multiple

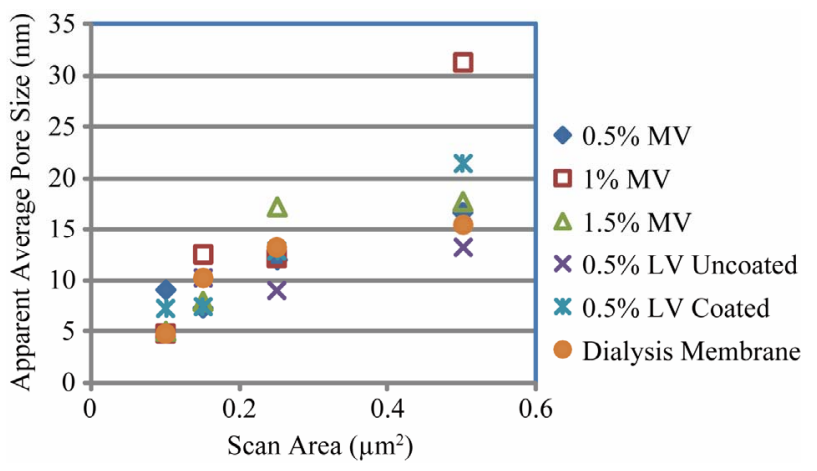

Figure 3. Variation in apparent pore size as a function of scan area.
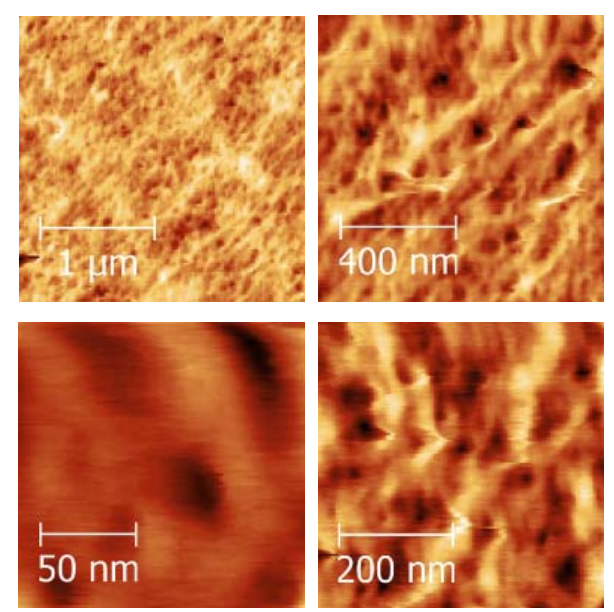

Figure 4. AFM images of $1.5 \% \mathrm{MV}$ alginate and $1.5 \%$ $\mathrm{CaCl}_{2}$. Clockwise from top left: 2.5, 1.0, 0.5 and $0.15 \mathrm{\mu m}^{2}$ scan area. 
Table 2. Effect of AFM scan area on apparent pore size.

\begin{tabular}{ccccccc}
\hline Sample & $\begin{array}{c}\mathrm{CaCl}_{2} \% \\
(\mathrm{w} / \mathrm{v})\end{array}$ & Coating & $\begin{array}{c}\text { Pore Size Range } \\
(\mathrm{nm}) \text { at } 0.5 \mu \mathrm{m}^{2}\end{array}$ & $\begin{array}{c}\text { Pore Size Range } \\
(\mathrm{nm}) \text { at } 0.25 \mu \mathrm{m}^{2}\end{array}$ & $\begin{array}{c}\text { Pore Size Range } \\
(\mathrm{nm}) \text { at } 0.15 \mu \mathrm{m}^{2}\end{array}$ & $\begin{array}{c}\text { Average Pore Size } \\
(\mathrm{nm}) \text { at } 0.1 \mu \mathrm{m}^{2}\end{array}$ \\
\hline Alginate MV 0.5\% (w/v) & 1.5 & $\mathrm{~N} / \mathrm{A}$ & $11-23$ & $7.0-16$ & $5.0-11$ & $8.4 \pm 3.0$ \\
Alginate MV 1.0\% (w/v) & 1.5 & $\mathrm{~N} / \mathrm{A}$ & $26-44$ & $9.0-16$ & $7.0-15$ & $4.5 \pm 1.1$ \\
Alginate MV 1.5\% (w/v) & 1.5 & N/A & $13-35$ & $12-28$ & $6.0-10$ & $5.2 \pm 0.9$ \\
Alginate LV 0.5\% (w/v) & 20 & N/A & $6.0-19$ & $6.0-12$ & $4.0-18$ & $7.2 \pm 2.9$ \\
Alginate LV 0.5\% (w/v) & 20 & Chitosan & $17-24$ & $7.0-19$ & $5.0-11$ & $7.0 \pm 3.1$ \\
Dialysis Tubing & N/A & N/A & $6.0-25$ & $6.0-14$ & $5.0-13$ & $4.9 \pm 3.0$ \\
\hline
\end{tabular}
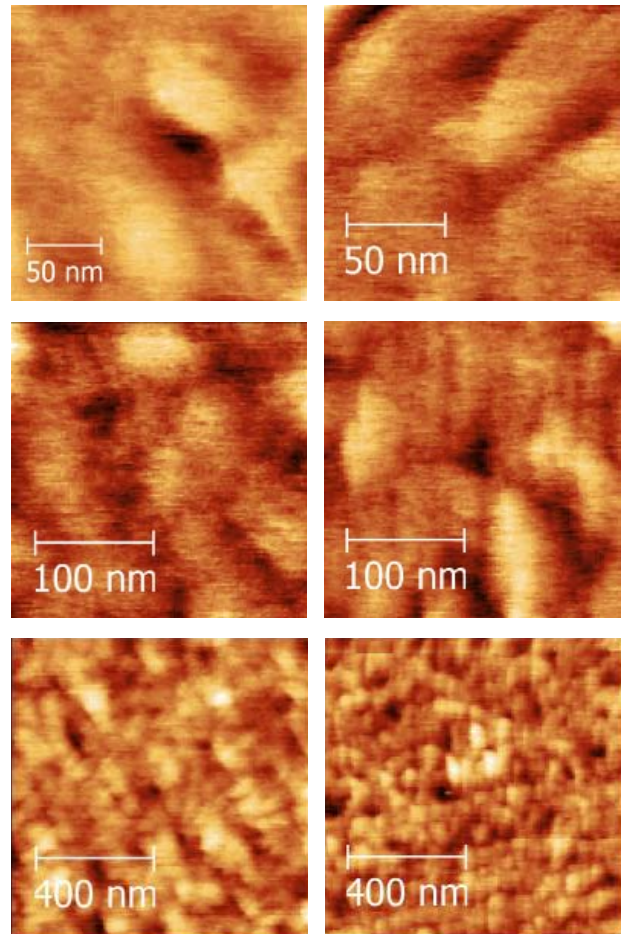

Figure 5. AFM images of $0.5 \% \mathrm{LV}$ alginate (left) and $0.5 \%$ LV alginate coated with chitosan (right): (top) Scan areas of $0.2 \mu^{2}$ (left) and $0.15 \mu^{2}$ (right); (middle) $0.25 \mu^{2}$ (left) and $0.25 \mu^{2}$ (right); (bottom): $1.0 \mu^{2}$ (left) and $1.0 \mu^{2}$ (right).

scan areas and a 3D view for the $0.1 \mu \mathrm{m}^{2}$ scan area for which the measured pore sizes are tabulated.

For the purposes of comparing AFM images, the following concentrations will be discussed: $1.5 \% \mathrm{MV} / 1.5 \%$ $\mathrm{CaCl}_{2}, 0.5 \% \mathrm{LV} / 20 \% \mathrm{CaCl}_{2}, 0.5 \% \mathrm{LV} / 20 \% \mathrm{CaCl}_{2}$ coated with $0.5 \%$ chitosan, and the dialysis membrane standard.

Higher scan areas produced what appear to be surfaces with deep, indented features, as indicated by the darkest areas of the images. MV alginate (Figure 4) and coated LV alginate (Figure 5) appear to have more defined surface features, which are most likely attributed to tipsample interaction due to the relative softness of the bare

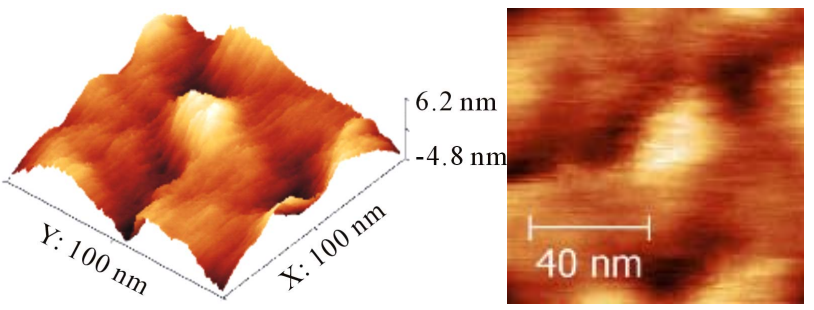

(a)

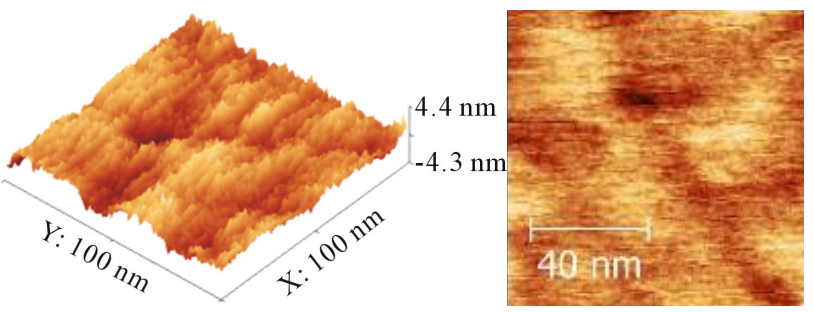

(b)

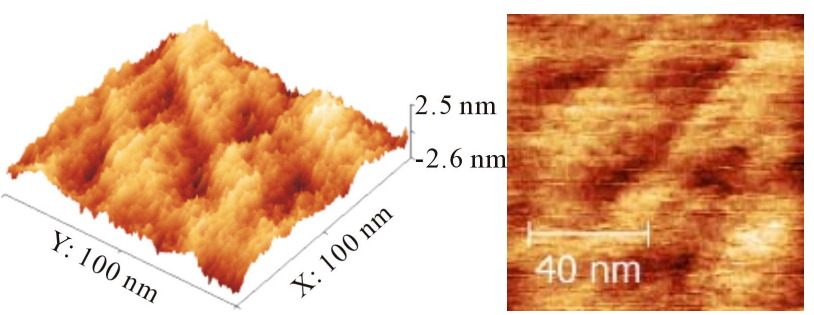

(c)

Figure 6. AFM 3D (left) and 2D (right) views of (a) $1.5 \%$ MV alginate, $0.1 \mathrm{\mu m}^{2}$ scan area; (b) $0.5 \% \mathrm{LV}$ alginate, 0.1 $\mu^{2}$ scan area; and (c) $0.5 \% \mathrm{LV}$ alginate coated with chitosan, $0.1 \mu^{2}$ scan area.

LV alginate. The layer of chitosan added to the LV alginate had some effect on film morphology. For example, chitosan-coated LV alginate displayed a structure with fewer features that were also of a smaller size than what was seen in the other films. This difference is due to the extra layer coating these features, effectively reducing the size of the pore openings.

As previously mentioned, it was observed that decreasing scan area also decreased the measured sizes of the pores. Since image resolution typically decreases as 

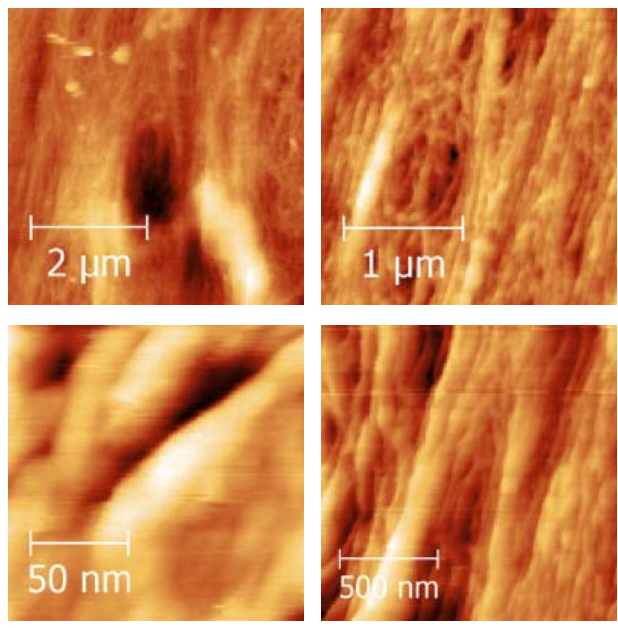

Figure 7. AFM images of dialysis tubing. Counterclockwise from top left: 5.0, 2.5, 1.5 and $0.15 \mu^{2}$ scan area.

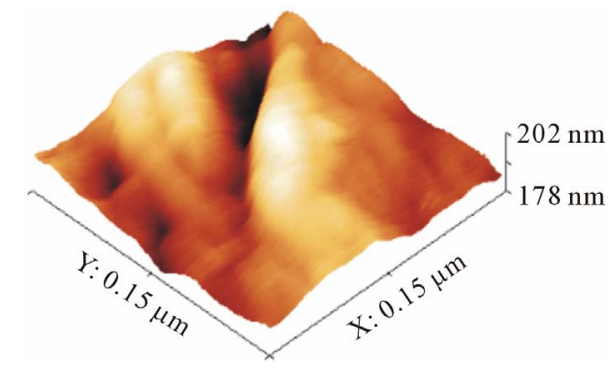

Figure 8. AFM 3D view of dialysis tubing, $0.15{\mu m^{2}}^{2}$ scan area.

scan size approaches $0.1 \mu \mathrm{m}^{2}$, this decrease in measured size appears counterintuitive. However, a decrease in scan area allows the ability to zoom into the deepest areas of the image, which represents the smallest opening of the pores as seen in the 3D images in Figure 6.

For AFM imaging of the dialysis tubing standard (Figure 7), the standard appears to have tolerated the AFM tip as indicated by the greater resolution and lower instance of artifacts across the surface compared to the alginate images. A 3D image of the dialysis tubing at 0.1 $\mu \mathrm{m}^{2}$ provided further means of visual comparison between a material of known porosity and the alginate porosity (Figure 8). The known porosity of the standard provided by the manufacturer lends feasibility to the use of the grayscale in estimating the pore size in the alginate material.

\subsection{Differential Scanning Calorimetry}

Shown in Figure 9 are sample DSC thermograms with corresponding glass transition temperatures presented in Table 3. Shown in the thermogram of sodium alginate (Sample A) is endothermic decay at $112^{\circ} \mathrm{C}$ due to removal of absorbed moisture (or nonstructural water). A
Table 3. Effect of cross-linker concentration on transition temperature.

\begin{tabular}{cccc}
\hline Sample & $\mathrm{CaCl}_{2} \%(\mathrm{w} / \mathrm{v})$ & Coating & $\mathrm{T}_{\mathrm{g}}\left({ }^{\circ} \mathrm{C}\right)$ \\
\hline A Alginate MV 1.5\%(w/v) & 0 & N/A & 112.81 \\
B Alginate MV 1.5\%(w/v) & 10 & N/A & 115.44 \\
C Alginate MV 1.5\%(w/v) & 15 & N/A & 115.42 \\
D Alginate MV 1.5\%(w/v) & 20 & N/A & 124.57 \\
E Alginate MV 1.5\%(w/v) & 25 & N/A & 127.77 \\
F Alginate MV 1.5\%(w/v) & 20 & Chitosan & 123.16 \\
G Alginate MV 1.5\% (w/v) & 25 & Chitosan & 135.68 \\
\hline
\end{tabular}

gradual increase in transition temperature and delayed endothermic shifts are observed with increasing $\mathrm{CaCl}_{2}$ concentration with an approximate step change of $9^{\circ} \mathrm{C}$ between the $15 \%$ - 20\% cross-linker concentration range (Samples C and D). Another marked increased is observed for sample $\mathrm{G}$ characterized by highest degree of cross-linking $\left(25 \%(\mathrm{w} / \mathrm{v}) \mathrm{CaCl}_{2}\right)$ and chitosan coating.

\subsection{Diffusivity Measurements}

Table 4 was generated by combining the results of diffusion experiments in MIs $(\mathrm{d}=40 \mu \mathrm{m} \pm 5 \mu \mathrm{m})$ and MAs $(\mathrm{d}$ $=1000 \mu \mathrm{m} \pm 75 \mu \mathrm{m}$ ) and AFM measurements. As shown in Figure 10, solute diffusivity is inversely correlated to $\lambda\left(\mathrm{R}^{2}=0.9835\right)$ and calculated in turn based on the AFM measurements. Assuming that the majority of pores are through pores, as the solute size approaches the pore size $(\lambda \geq 1)$, the solute cannot be filtered through the membrane.

Reflection coefficients equal to or exceeding unity indicate the lack of membrane permeability to the specific solute as reflected by the $10^{3}-10^{4}$ fold reduction in diffusivity values as the marker MW was increased.

\section{Discussion}

\subsection{Atomic Force Microscopy}

In terms of variability in pore measurement, the darkest areas using grayscale intensity were used to measure pore size. This method effectively used the smallest opening as the pore width. Although measurements of dialysis tubing AFM images using Gwyddion analysis tools yielded an average pore size of $4.9 \mathrm{~nm}$, the actual pore size could not be definitively measured to less than $7-10$ nm due to the manufacturer's specification on the tip radius. However, based on consistently-measured dialysis tubing pores using grayscale intensity, the trend clearly indicated that the ability to resolve the pores increased with decreasing scan size. As the scan size de- 


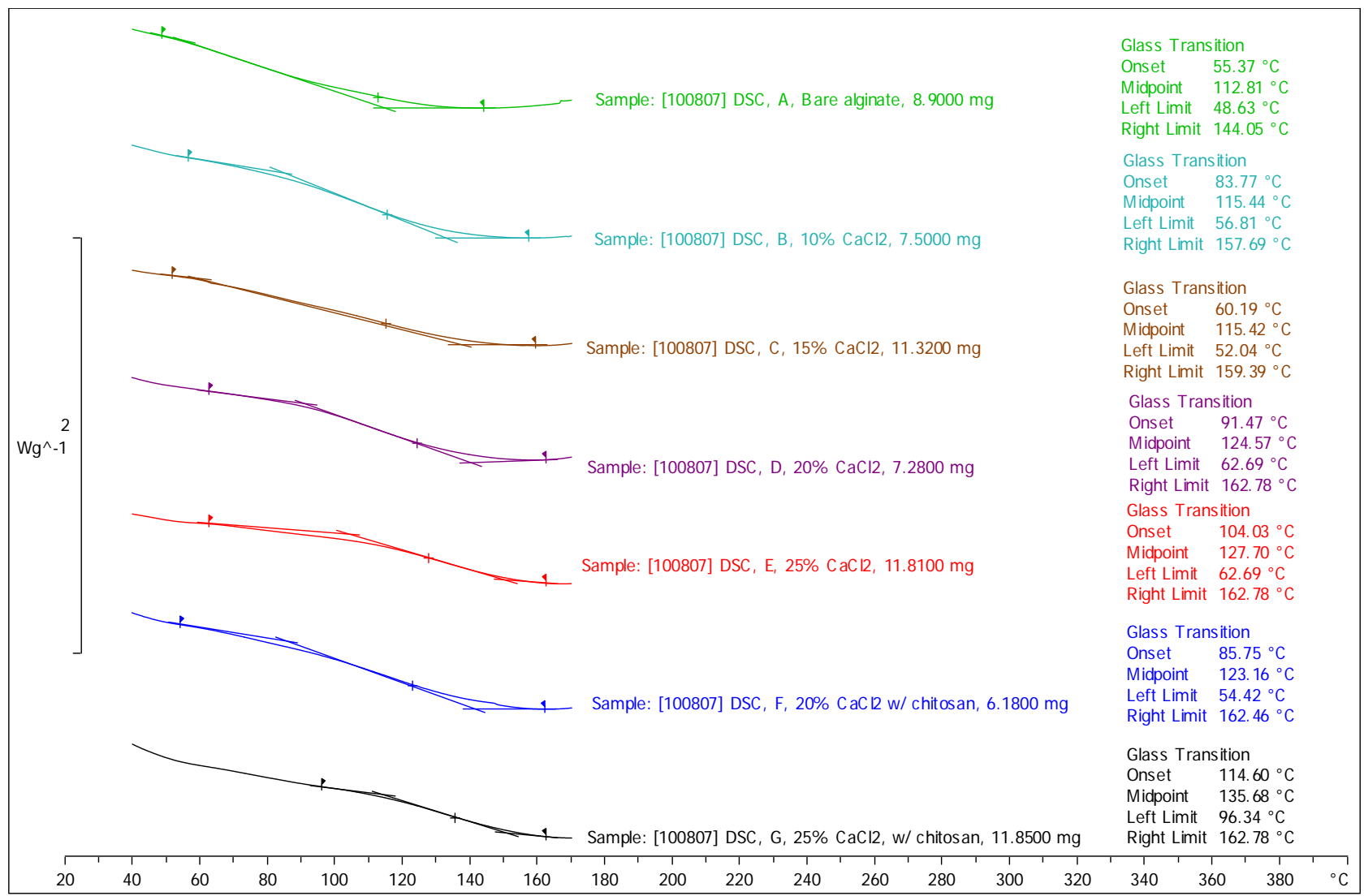

Figure 9. DSC thermograms with corresponding glass transition temperatures.

Table 4. Results of membrane diffusivity, pore size and reflection coefficient across different formulations and molecular weight markers.

\begin{tabular}{|c|c|c|c|c|c|c|c|c|}
\hline Membrane & $\mathrm{CaCl}_{2} \%(\mathrm{w} / \mathrm{v})$ & Coating & Measurement Method & MW Marker & $\mathrm{a}(\mathrm{nm})$ & $\mathrm{r}(\mathrm{nm})$ & $\sigma$ & $\mathrm{D}\left(\mathrm{m}^{2} / \mathrm{s}\right)$ \\
\hline Alginate MV $1.5 \%(\mathrm{w} / \mathrm{v})$ & 1.5 & N/A & Spectrophotometry & Creatinine & 0.36 & 2.6 & 0.4 & $7.20 \mathrm{E}-13$ \\
\hline Alginate LV $0.5 \%(\mathrm{w} / \mathrm{v})$ & 20 & Chitosan & Fluorescence Microscopy & FITC dextran $4 \mathrm{kDa}$ & 1.18 & 3.5 & 0.4 & $7.70 \mathrm{E}-14$ \\
\hline Alginate MV $1.5 \%(\mathrm{w} / \mathrm{v})$ & 1.5 & N/A & Fluorescence Microscopy & FITC dextran $4 \mathrm{kDa}$ & 1.18 & 2.6 & 0.5 & $1.81 \mathrm{E}-14$ \\
\hline Alginate LV $0.5 \%(\mathrm{w} / \mathrm{v})$ & 20 & Chitosan & Fluorescence Microscopy & FITC dextran $70 \mathrm{kDa}$ & 3.07 & 3.5 & 1.0 & $3.02 \mathrm{E}-16$ \\
\hline Alginate MV $1.5 \%(\mathrm{w} / \mathrm{v})$ & 1.5 & N/A & Fluorescence Microscopy & FITC dextran $70 \mathrm{kDa}$ & 3.07 & 2.6 & 1.0 & $5.95 \mathrm{E}-17$ \\
\hline Alginate MV $1.5 \%(\mathrm{w} / \mathrm{v})$ & 1.5 & $\mathrm{~N} / \mathrm{A}$ & Fluorescence Microscopy & FITC dextran $500 \mathrm{kDa}$ & 5.92 & 2.6 & 2.6 & 0 \\
\hline Alginate LV $0.5 \%(\mathrm{w} / \mathrm{v})$ & 20 & Chitosan & Fluorescence Microscopy & FITC dextran $500 \mathrm{kDa}$ & 5.92 & 3.5 & 1.0 & 0 \\
\hline
\end{tabular}

creased, the measured and calculated Stokes' radius of the tubing began to converge, and these results lend confidence into this method of measurement. However, for the purposes of this study, the average pore size of 4.9 $\mathrm{nm}$ at a scan size of $0.1 \mu \mathrm{m}^{2}$ is a relative measurement and requires further investigation with a tip of smaller radius or alternate imaging method.

SEM facilities were available for this study; however, the equipment did not provide the desired resolution for pore measurements, with a limit of $100 \mathrm{~nm}$ on the given system. In addition, radiation generated by the SEM electron beam is known to cause cross-linking, which would have required further study in terms of potential effect on the alginate $/ \mathrm{CaCl}_{2}$ porosity. Transmission electron microscopy (TEM) has been used to image alginate in previous studies [26]; however sample preparation methods for both SEM and TEM include a number of fixing media including glutaraldehyde [10,26,35], the primary function of which is to provide structure by cross-linking biological materials prior to dehydration, 


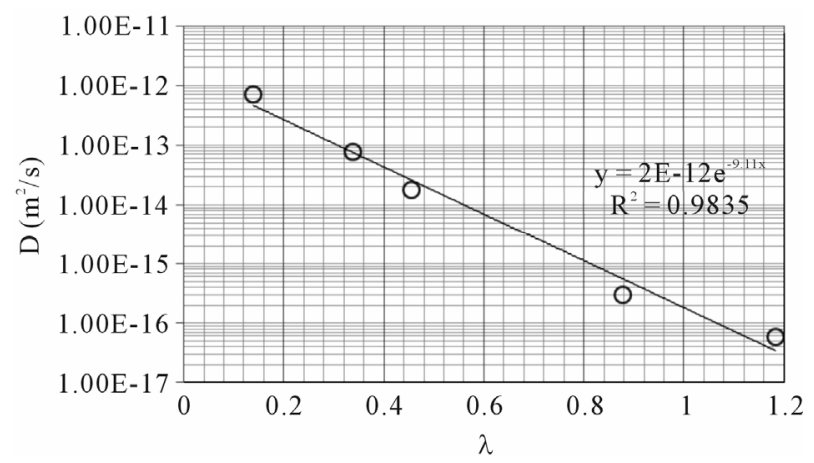

Figure 10. Membrane diffusivity as a function of solute to membrane pore size.

where changes to cross-linking in the alginate would not be desired. If fixation were not an issue with the alginate/ $\mathrm{CaCl}_{2}$, SEM and TEM samples would then be dehydrated after fixation using critical point drying [35] or lyophilization [36] and coated with a conductive coating or imaged in low-vacuum SEM without a conductive coating; however, SEM images of samples prepared using these dehydration methods clearly indicate damage and shrinkage of the material [26] and are not a desired sample preparation method. Alcohol or acetone substitution could have been used with environmental SEM (ESEM) [35]; however, a cold stage was not available. A cold stage allows a hydrated sample to remain at the dew point in the SEM chamber. By varying the temperature or pressure in the chamber, the user can cause the sample to dehydrate in a controlled fashion so that some of the surface moisture sublimates but without completely drying the surface, where with a wet surface, the SEM electron beam would image the liquid instead of the sample surface. Lastly, scanning tunneling microscopy (STM) offers Ångström resolution; however, dehydration or fixing of the sample would have been required in order to apply a conductive coating on the sample for STM. Therefore, imaging in the native state using AFM with minimal sample preparation was preferable.

Intermittent contact or tapping mode with the sample fully immersed in liquid generally reduces the likelihood of surface damage by the probe tip; however, in this study, intermittent contact mode did not provide the desired resolution. This problem may have been due to the stickiness of the sample interacting with the tip [26]. It was determined that the sample could be sufficiently imaged by AFM using contact mode with a low stiffness probe of $0.08 \mathrm{~N} / \mathrm{m}$ to reduce damage to the surface. Other groups were found to have performed AFM imaging using a higher stiffness probe such as $0.12 \mathrm{~N} / \mathrm{m}[27$, 37]. In the case of the $0.5 \% \mathrm{LV}$ alginate concentration, the initial samples were too soft for AFM imaging and required an increase in cross-linking concentration to stiffen the material.

\subsection{Calorimetry}

Using ionotropic gelation by which all cross-linked samples have been fabricated, at a given initial alginate concentration, the degree of cross-linking can be varied by either modulating the $\mathrm{CaCl}_{2}$ concentration or modifying the $\mathrm{G}$ block content of the bio-polymer. The DSC analysis that was performed revealed that increasing the cross-linker concentration resulted in an increase in glass transition temperature $\left(\mathrm{T}_{\mathrm{g}}\right)$.

The increase in $T_{g}$ can be attributed to the linking that could restrict the molecular response to temperature change as predicted by classical polymer theory [38]. As would be expected, higher $\mathrm{CaCl}_{2}$ concentrations have a more pronounced effect on free volume as an increase in $\mathrm{CaCl}_{2}$ ions provide more opportunities for creating tie points between polymer chains. Recent results of thermogravimetric (TGA) analysis on alginate films confirmed the same trend [39]. It is known from the literature that, there are three kinds of absorbed water in hydrophilic polymers [40,41], free, freezing bound, and non-freezing bound. Whereas freezing bound water interacts weakly, non-freezing bound water forms hydrogen bond to bind with the polymeric chain. As stated in the methodology section, since care was taken to remove the free water by desiccation, and alginate [42] and chitosan [43] decomposition occur at temperatures above $200^{\circ} \mathrm{C}$, it could be hypothesized that the shifts in transition temperatures detected are due to the elimination of the freezing and non-freezing bound water. As for the increase in $T_{g}$, as a result of the chitosan coating at higher cross-linking concentrations, chitosan is classified as a stiff and rigid polyelectrolyte. Once adsorbed onto the bio-membrane, a more rigid and less fluid bio-membrane characterized by higher glass transition temperatures has been reported $[44,45]$. The results contradict findings of Russo, Malinconico and Santagata. In that study, an increase in the guluronic acid content of the alginate resulted in a decrease in glass transition temperatures measured by DSC. A higher G block content resulted in swelling and lower $\mathrm{T}_{\mathrm{g}}$ for the cross-linked hydrogel. The authors hypothesize that, as expected, the cross-linking points represent a hindrance for the packing of chains; however, the chain segments between two consecutive cross-linking points experience an increased mobility because of the increase of the free volume due to swelling.

\subsection{Diffusivity Measurements}

Whether using spectrophotometry or fluorescence microscopy for diffusivity determination, experiments were 
designed to avoid the following interactions affecting pore size measurements: 1) the capsules had reached an equilibrium swollen state post-fabrication monitored by microscopy; 2) the MW markers chosen for the graph do not react with the pores; 3 ) the MW markers did not react with the membrane using electrostatic and hydrophobic interactions; 4) the solute was not present in excess at the membrane interface to generate concentration polarization except for the $500 \mathrm{kDa}$ marker to which the membrane is impermeable [46]; and 5) multiple sources place the $70 \mathrm{kDa}$ marker at the MW cutoff of the membrane $[33,46]$ so diffusion was not hindered for creatinine or the $4 \mathrm{kDa}$ marker. Given these precautions and the diffusivity measurements, it could be hypothesized that a portion of the detected pores by AFM are through pores.

\section{Conclusions}

The surface morphology of cross-linked alginate structures was investigated through the use of DSC, AFM and diffusivity measurements using spectrophotometry and fluorescence microscopy. Through DSC measurements, successful cross-linking was established by correlating glass transition temperature and cross-linker concentration. AFM experiments performed on alginate films yielded pore sizes for $1.5 \% \mathrm{CaCl}_{2}$ and $0.5 \%(\mathrm{w} / \mathrm{v})$ alginate/chitosan cross-linked with $20 \% \mathrm{CaCl}_{2}$ to be $5.2 \mathrm{~nm}$ $\pm 0.9 \mathrm{~nm}$ and $7.0 \mathrm{~nm} \pm 3.1 \mathrm{~nm}$, respectively. Through measurements of inward diffusivity and outward diffusivity of MW marker, the presence of through pores in the alginate membrane was confirmed. Decreasing diffusivities and reflection coefficients approaching unity concur with previous findings that the molecular weight cutoff of the studied alginate bio-membranes is approximately $70 \mathrm{kDa}$.

Since it is difficult to confirm the accuracy of measuring pore sizes through the grayscale intensity method, the following improvements should be considered: 1) scan size should start at $0.1 \mu \mathrm{m}^{2}$, and finer AFM probe tips should be investigated such as molecularly-functionalized tips; 2) the number of pixels/line should be increased to improve resolution due to a slower scan rate; 3 ) the use of liquid imaging with tapping or intermittent contact mode should be re-evaluated, and a lower stiffness probe should be used to further reduce damage to the soft sample surface; and 4) high resolution field effect SEM (FESEM) imaging [26] to the scale of $1-5 \mathrm{~nm}$ resolution [15] could be used in future work to quantify differences in pore size or shape between the microcapsules and films if the material can be prepared for SEM without additional damage to the material from sample preparation.

This further understanding of alginate morphology can potentially be helpful in determining how to fine-tune alginate pore sizes and to carefully regulate release kinetics from alginate membranes.

\section{Acknowledgements}

The authors would like to acknowledge the Davidson's College of Engineering Faculty Development ("Inkjet Bioprinting") and Junior Professorship Grants, the CSUPERB Joint Venture Grant "Bio-Printing of Mammalian Cells", and the C-SUPERB Faculty-Student Collaborative Research/New Investigator Grant Program "Encapsulated Hybrid P450 enzyme as novel light-driven biocatalyst" for funding this effort. Thermal characterization of the samples could not have been executed without access to the DSC machine granted generously by BAE Systems, San Jose, CA.

\section{REFERENCES}

[1] T. R. Hoare and D. S. Kohane, "Hydrogels in Drug Delivery: Progress and Challenges," Polymer, Vol. 49, No. 8, 2008, pp. 1993-2007. http://dx.doi.org/10.1016/j.polymer.2008.01.027

[2] T. M. S. Chang, "Artificial Cells: Biotechnology, Nanomedicine, Regenerative Medicine, Blood Substitutes, Bioencapsulation and Cell/Stem Cell Therapy-Vol. 1," World Scientific Publishing Co. Pte. Ltd., Singapore City, 2007.

[3] H. Li, "Smart Hydrogel Modelling," Springer, Singapore City, 2009. http://dx.doi.org/10.1007/978-3-642-02368-2

[4] A. Paul, G. Chen, A Khan, V. T. Rao, D. Shum-Tim and S. Prakash, "Genipin-cross-linked Microencapsulated Human Adipose Stem Cells Augment Transplant Retention Resulting in Attenuation of Chronically Infarcted Rat Heart Fibrosis and Cardiac Dysfunction," Cell Transplantation, Vol. 21, No. 12, 2012, pp. 2735-2751. http://dx.doi.org/10.3727/096368912X637497

[5] M. Sridhar-Keralapura, S. Thirumalai and M. MobedMiremadi, "Structural Changes and Imaging Signatures of Acoustically Sensitive Microcapsules under Ultrasound," Ultrasonics, Vol. 53, No. 25, 2013, pp. 10441057. http://dx.doi.org/10.1016/j.ultras.2013.02.001

[6] J. Zhu and R. E. Marchant, "Design Properties of Hydrogel Tissue-engineering Scaffolds," Expert Review of Medical Devices, Vol. 8, No. 5, 2011, pp. 607-626. http://dx.doi.org/10.1586/erd.11.27

[7] N. Wang, G. Adams, L. Buttery, F. H. Falcone and S. Stolnik, "Alginate Encapsulation Technology Supports Embryonic Stem Cell," Journal of Biotechnology, Vol. 144, No. 4, 2009, pp. 304-312. http://dx.doi.org/10.1016/j.jbiotec.2009.08.008

[8] I. Braccini, R. P. Grasso and S. Pérez, "Conformational and Configurational Features of Acidic Polysaccharides and Their Interactions with Calcium Ions: A Molecular Modeling Investigation," Carbohydrate Research, Vol. 317, No. 1, 1999, pp. 119-130.

http://dx.doi.org/10.1016/S0008-6215(99)00062-2 
[9] H. Uludag, P. De Vos and P. A. Tresco, "Technology of Mammalian Cell Encapsulation," Advanced Drug Delivery Reviews, Vol. 42, No. 1, 2000, pp. 29-64. http://dx.doi.org/10.1016/S0169-409X(00)00053-3

[10] H. Zimmermann, F. Wählisch, C. Baier, N. Westhoff, R. Reuss, D. Zimmermann, M. Behringer, F. Ehrhart, A. Katsen-Globa, C. Giese, U. Marx, V. L. Sukhorukov, J. A. Vásquez, P. Jakob, S. G. Shirley and U. Zimmermann, "Physical and Biological Properties of Barium Crosslinked Alginate Membranes," Biomaterials, Vol. 28, No. 7, 2006, pp. 1327-1345. http://dx.doi.org/10.1016/j.biomaterials.2006.11.032

[11] H. Chen, W. Ouyang, B. Lawuyi and S. Prakash, "Genipin Cross-Linked Alginate-Chitosan Microcapsules: Membrane Characterization and Optimization of Cross-Linking Reaction," Biomacromolecules, Vol. 7, No. 7, 2006, pp. 2091-2098. http://dx.doi.org/10.1021/bm050862y

[12] A. Tamayol, M. Akbari, N. Annabi, A. Paul, A. Khadamhosseini and D. Juncker, "Fiber-Based Tissue Engineering: Progress, Challenges, and Opportunities," Biotechnology Advances, Vol. 31, No. 5, 2012, pp. 669-687. http://dx.doi.org/10.1016/j.biotechadv.2012.11.007

[13] M. Mobed-Miremadi, "High Throughput Methods for Miniaturization of Implantable Artificial Cells," In: T. M. S. Chan, Ed., Selected Topics in Nanomedicine: Regenerative Medicine, Artificial Cells and Nanomedicine-Vol. 3, World Scientific Publishing Co. Pte. Ltd., Singapore City, in Press.

[14] Y. K. Demir, Z. Akan and O. Kerimoglu, "Sodium Alginate Microneedle Arrays Mediate the Transdermal Delivery of Bovine Serum Albumin," PLoS ONE, Vol. 8, No. 5, 2013, Article ID: e63819. http://dx.doi.org/10.1371/journal.pone.0063819

[15] W. R. Gombotz and S. Wee, "Protein Release from Alginate Matrices," Advanced Drug Delivery Reviews, Vol. 31, No. 3, 1998, pp. 267-285. http://dx.doi.org/10.1016/S0169-409X(97)00124-5

[16] M. A. J. Mazumder, N. A. D. Burke, F. Shen, T. Chu, M. A. Potter and H. D. H. Stover, "Synthetic Reactive Polyelectrolytes for Cell Encapsulation," ACS Symposium Series, Oxford University Press, Cary, 2010, pp. 131-159. http://dx.doi.org/10.1021/bk-2010-1053.ch007

[17] R. Russo, M. Malinconico and G. Santagata, "Effect of Cross-Linking with Calcium Ions on the Physical Properties of Alginate Films," Biomacromolecules, Vol. 8, No. 10, 2007, pp. 3193-3197. http://dx.doi.org/10.1021/bm700565h

[18] S. Ahn, H. Lee, L. J. Bonassar and G. Kim, "Cells (MC3T3E1)-Laden Alginate Scaffolds Fabricated by a Modified Solid-Freeform Fabrication Process Supplemented with an Aerosol Spraying," Biomacromolecues, Vol. 13, No. 9, 2012, pp. 2997-3003. http://dx.doi.org/10.1021/bm3011352

[19] R. L. Fournier, "Basic Transport Phenomena in Biomedical Engineering," 3rd Edition, Taylor \& Francis, Philadelphia, 2011.

[20] S. Amin, S. Rajabnezhad and K. Kohli, "Hydrogels as
Potential Drug Delivery Systems," Scientific Research and Essay, Vol. 3, No. 11, 2011, pp. 1175-1183.

[21] N. W. Choi, M. Cabodi, B. Held, J. P. Gleghorn, L. J. Bonassar and A. D. Strook, "Microfluidic Scaffolds for Tissue Engineering," Nature Materials, Vol. 6, No. 11, 2007, pp. 908-915. http://dx.doi.org/10.1038/nmat2022

[22] A. W. Chan and R. J. Neufeld, "Tuneable Semi-Synthetic Network Alginate for Absorptive Encapsulation and Controlled Release of Protein Therapeutics," Biomaterials, Vol. 31, No. 34, 2010, pp. 9040-9047. http://dx.doi.org/10.1016/j.biomaterials.2010.07.111

[23] R. H. Li, D. H. Altreuter and F. T. Gentile, "Transport Characterization of Hydrogel Matrices for Cell Encapsulation," Biotechnology and Bioengineering, Vol. 50, No. 4, 1996, pp. 365-373. http://dx.doi.org/10.1002/(SICI)1097-0290(19960520)50: 4<365::AID-BIT3>3.0.CO;2-J

[24] B. Wright, R. A. Cave, J. P. Cook, V. V. Khutoryansky, S. Mi, B. Chen, M. Leyland and C. J. Connon, "Enhanced Viability of Corneal Epithelial Cells for Efficient Transport/storage Using a Structurally Modified Calcium Alginate Hydrogel," Regenerative Medicine, Vol. 7, No. 3, 2012, pp. 295-307. http://dx.doi.org/10.2217/rme.12.7

[25] A. Jejurikar, G. Lawrie, D. Martin and L. Grøndahl, “A Novel Strategy for Preparing Mechanically Robust Ionically Cross-Linked Alginate Hydrogels," Biomedical Materials, Vol. 6., No. 2, 2011, Article ID: 025010. http://dx.doi.org/10.1088/1748-6041/6/2/025010

[26] S. X. Hsiong, P. H. Cooke, H. Kong, M. L. Fishman, M. Ericsson and D. J. Mooney, "AFM Imaging of RGD Presenting Synthetic Extracellular Matrix Using Gold Nanoparticles," Macromolecular Bioscience, Vol. 8, No. 6, 2008, pp. 469-477.

http://dx.doi.org/10.1002/mabi.200700313

[27] T. Schmid, J. Burkhard, B. Yeo, W. Zhang and R. Zenobi, "Towards Chemical Analysis of Nanostructures in Biofilms I: Imaging of Biological Nanostructures," Analytical and Bioanalytical Chemistry, Vol. 391, No. 5, pp 1899-1905. http://dx.doi.org/10.1007/s00216-008-2100-2

[28] A. Leal-Egaña, U. Braumann, A. Díaz-Cuenca, M. Nowicki and A. Bader, "Determination of Pore Size Distribution at the Cell-hydrogel Interface," Journal of Nanobiotechnology, Vol. 9, No. 24, 2011, pp. 1-7. http://dx.doi.org/10.1186/1477-3155-9-24

[29] M. Mobed-Miremadi, B. Asi, J. Parasseril, E. Wong, M. Tat and Y. Shan, "Comparative Diffusivity Measurements for Alginate-Based Atomized and Inkjet-Bioprinted Artificial Cells Using Fluorescence Microscopy," Artificial Cells, Nanomedicine, and Biotechnology, Vol. 41 No. 3, 2013, pp. 196-201. http://dx.doi.org/10.3109/10731199.2012.716064

[30] M. Mobed-Miremadi and S. Dharba, "Immobilization of R. Erythropolis in Alginate-Based Artificial Cells for Simulated Plaque Degradation in Aqueous Media," Taylor \& Francis, Philadelphia. http://dx.doi.org/10.3109/02652048.2013.814726

[31] S. Prakash and T. M. S. Chang, "Preparation and In-Vitro 
Analysis of Genetically Engineered E. coli DH5 Cells Microencapsulated in Artificial Cells for Urea and Ammonia Removal," Biotechnology and Bioengineering, Vol. 46, No. 6, 1995, pp. 621-626.

http://dx.doi.org/10.1002/bit.260460615

[32] M. Mobed-Miremadi, E. Acks, S. Polsaward and D. Chen, "High Throughput Miniaturization of Artificial Cells," Artificial Cells, Nanomedicine, and Biotechnology, Vol. 39, No. 5, 2011, pp. 310-316. http://dx.doi.org/10.3109/10731199.2011.574637

[33] M. F. A. Goosen, "Fundamentals of Animal Cell: Encapsulation and Immobilization," CRC Press, Boca Raton, 1993.

[34] Y. Shan, "Viability of Microencapsulation of E. coli DH5 for Urea Removal Using Inkjet Bio-Printing," Masters Project Report, San Jose State University, San Jose, 2011. http://generalengineering.sjsu.edu/docs/pdf/mse_prj_rpts/ fall2011/VIABILITY_OF_MICROENCAPSULATION_ OF_E.COLI_DH5_FŌR_ÜREA.pdf

[35] L. Muscariello, F. Rosso, G. Marino, A. Giordano, M. Barbarisi, G. Cafiero and A. Barbarisi, "A Critical Overview of ESEM Applications in the Biological Field," Journal of Cellular Physiology, Vol. 205, No. 3, 2005, pp. 328-334. http://dx.doi.org/10.1002/jcp.20444

[36] M. Rubert, M. Monjo, S. P. Lyngstadaas and J. M. Ramis, "Effect of Alginate Hydrogel Containing PolyprolineRich Peptides on Osteoblast Differentiation," Biomedical Materials, Vol. 7, No. 5, 2012, Article ID: 055003. http://dx.doi.org/10.1088/1748-6041/7/5/055003

[37] Z. Keresztes, T. Rigo, J. Telegdi and E. Kalman, "Investigation of Biopolymer Networks by Means of AFM," Applied Physics A, Materials Science \& Processing, Vol. 72, Suppl. 1, 2001, pp. S113-S116. http://dx.doi.org/10.1007/s003390100680

[38] S. K. H. Gulrez, S. Al-Assaf and G. O. Phillips, "Hydrogels: Methods of Preparation, Characterisation and Appliation," In: A. Carpi, Ed., Progress in Molecular and Environmental Bioengineering-From Analysis and Modeling to Technology Applications, Intech Europe, Croatia, 2011, pp. 117-150.

http://www.intechopen.com/books/progress-in-molecular and-environmental-bioengineering-from-analysis-and-mo delng-to-technology-applications/hydrogels-methods-of-p reparation-characterisation-and-applications
[39] V. Urquiza, K. Turner, O. Perales Pérez and M. Gálvez Saldaña, "Effect of the Cross-Linking with Calcium Ions on the Structural and Thermo-Mechanical Properties of Alginate Films," MRS Proceedings, Vol. 1355, No. 1, Cambridge University Press, Cambridge, 2011. http://dx.doi.org/10.1557/opl.2011.1136

[40] S. J. Kim, S. G. Yoon and S. I. Kim, "Synthesis and Characteristics of Interpenetrating Polymer Network Hydrogels Composed of Alginate and Poly (Diallydimethylammonium Chloride)," Journal of Applied Polymer Science, Vol. 91, No. 6, 2004, pp. 3705-3709. http://dx.doi.org/10.1002/app.13615

[41] K. Nakamura, T. Hatakeyama and H. Hatakeyama, "Relationship between Hydrogen Bonding and Bound Water in Polyhydroxystyrene Derivatives," Polymer, Vol. 24, No. 7, 1983, pp. 871-876. http://dx.doi.org/10.1016/0032-3861(83)90206-9

[42] T. S. Pathak, J. H. Yun, J. Lee and K. J. Paeng, "Effect of Calcium Ion (Cross-Linker) Concentration on Porosity, Surface Morphology and Thermal Behavior of Calcium Alginates Prepared from Algae (Undaria pinnatifida)," Carbohydrate Polymers, Vol. 81, No. 3, 2010, pp. 633-639. http://dx.doi.org/10.1016/j.carbpol.2010.03.025

[43] S. Shabbear, S. Ramanamurthy and K. V. Ramanamurthy, "Formulation and Evaluation of Chitosan Sodium Alginate Microcapsules of 5-Fluorouracil for Colorectal Cancer," International Journal of Research in Pharmacy and Chemistry, Vol. 2, No. 1, 2012, pp. 7-19. http://dx.doi.org/10.1186/2194-0517-2-1

[44] S. J. Florczyk, D. J. Kim, D. L. Wood and M. Zhang, "Influence of Processing Parameters on Pore Structure of 3D Porous Chitosan-Alginate Polyelectrolyte Complex Scaffolds," Journal of Biomedical Materials Research Part A, Vol. 98, No. 4, 2011, pp. 614-620. http://dx.doi.org/10.1002/jbm.a.33153

[45] J. Shaji, V. Jain and S. Lodha, "Chitosan: A Novel Pharmaceutical Excipient," International Journal of Pharmaceutical Excipient and Applied Sciences, Vol. 1, No. 1, 2010, pp. 11-28.

[46] S. Prakash and C. Martoni, "Toward a New Generation of Therapeutics," Applied Biochemistry and Biotechnology, Vol. 128, No. 1, 2006, pp. 1-22. http://dx.doi.org/10.1385/ABAB:128:1:001 